\title{
Rapid Prototyping Human Interfaces Using Stretchable Strain Sensor
}

\author{
Tokiya Yamaji, ${ }^{1}$ Hiroyuki Nakamoto, ${ }^{1}$ Hideo Ootaka, ${ }^{2}$ \\ Ichiro Hirata, ${ }^{3}$ and Futoshi Kobayashi ${ }^{1}$ \\ ${ }^{1}$ Kobe University, 1-1 Rokkodaicho, Nada, Kobe 657-8501, Japan \\ ${ }^{2}$ Bando Chemical Industries, 4-6-6 Minatojima Minamimachi, Chuo, Kobe 650-0047, Japan \\ ${ }^{3}$ Hyogo Prefecture Institute of Technology, 3-1-12 Yukihiracho, Suma, Kobe 654-0037, Japan \\ Correspondence should be addressed to Hiroyuki Nakamoto; nakamoto@panda.kobe-u.ac.jp
}

Received 5 August 2016; Accepted 29 November 2016; Published 2 January 2017

Academic Editor: Chenzhong Li

Copyright (C) 2017 Tokiya Yamaji et al. This is an open access article distributed under the Creative Commons Attribution License, which permits unrestricted use, distribution, and reproduction in any medium, provided the original work is properly cited.

In the modern society with a variety of information electronic devices, human interfaces increase their importance in a boundary of a human and a device. In general, the human is required to get used to the device. Even if the device is designed as a universal device or a high-usability device, the device is not suitable for all users. The usability of the device depends on the individual user. Therefore, personalized and customized human interfaces are effective for the user. To create customized interfaces, we propose rapid prototyping human interfaces using stretchable strain sensors. The human interfaces comprise parts formed by a threedimensional printer and the four strain sensors. The three-dimensional printer easily makes customized human interfaces. The outputs of the interface are calculated based on the sensor's lengths. Experiments evaluate three human interfaces: a sheet-shaped interface, a sliding lever interface, and a tilting lever interface. We confirm that the three human interfaces obtain input operations with a high accuracy.

\section{Introduction}

In the modern society with a variety of information electronic devices, human interfaces increase their importance in a boundary of a human and a device. In general, the human is required to get used to the device. Even if the device is designed as a universal device or a high-usability device, the device is not suitable for all users. The usability of the device depends on the individual user. Therefore, personalized and customized human interfaces are effective for the user. Recently, there are some papers with regard to personalized human interfaces. Kimura and Yamane used virtual product models to design human operational interfaces [1]. Ha et al. proposed a haptic prototyping system with a motoractuated dial knob [2]. Their approaches aimed at design of common and high-usability interfaces. Yau et al. evaluated input devices for cursor-positioning tasks [3]. Turpin et al. evaluated computer input devices for people with disabilities and reported needs of customized interfaces [4]. Savage et al. proposed a system to assist designers creating human interface using a three-dimensional printer [5]. The customized interfaces improve working efficiency and help in using of devices for people with disability. To make the customized interfaces, technical foundations are being ready. One of the foundations is a three-dimensional printer (hereinafter referred to as "3D printer"). The 3D printer forms parts based on digital models and is effective for making parts of human interfaces. In addition, the cost of the 3D printer has gone down lately. Another of the technical foundations is a flexible sensor to obtain the motion of the parts.

Flexible and stretchable electronics have been developed. This technique applies elasticity to electronics. Developments advance from passive elements such as substrates, cables, and LEDs to functional elements such as actuators and sensors [6-10]. Application to various fields is considered using flexible and stretchable electronics, for example, production of an artificial muscle using electroactive macromolecule [11], development of artificial skin for robots using a flexible 


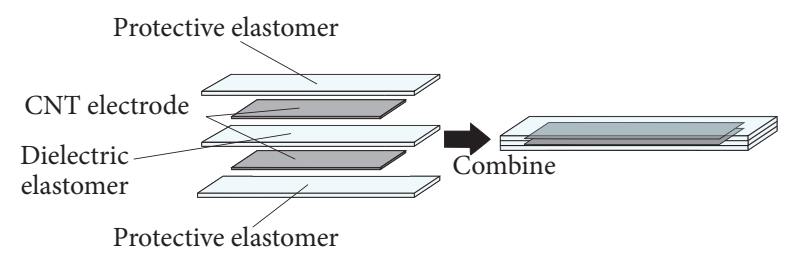

FIGURE 1: Structure of stretchable strain sensor.

sense of touch sensor [12], and measurement of the human knee's angle [13]. Flexible and stretchable sensors have been reported. Lipomi et al. proposed a strain sensor using the carbon nanotubes' electrodes [14]. In addition, Sato et al. reported various gesture detection using the sensing technology which enabled gesture input by measuring capacitance in a widespread frequency band [15]. We also proposed a stretchable strain sensor. The strain sensor is thin and lightweight and stretches up to $200 \%$ [16]. The combination of the 3D printer and the flexible sensor provides rapid prototyping human interfaces. The shape and size of the interfaces are easily changeable for users' needs. In this study, rapid prototyping human interfaces using a stretchable strain sensor are proposed. A 3D printer forms parts of interfaces. The motion of the constructed interfaces is measured by the strain sensors. The strain sensors are attached to the interfaces and measure the distances between the parts. The outputs of the interfaces are calculated based on the distances. Three kinds of interfaces are evaluated through laboratory experiments. It is confirmed that the interfaces using the $3 \mathrm{D}$ printer and the strain sensor are effective.

\section{Stretchable Strain Sensor}

2.1. Structure. The stretchable strain sensor has three thin sheets of elastomer and two stretchable electrodes. Figure 1 shows the structure of the stretchable strain sensor. The three elastic sheets sandwich the two electrode sheets. About the layers of the elastomer, the top and bottom layers have a role to protect electrodes. The middle layer has a role to be a dielectric layer. The elastomer is flexible and light weight. The thickness of the three-layer stretchable strain sensor is approximately $150 \mu \mathrm{m}$. The electrodes are made from carbon nanotubes [16]. The thickness of the electrode is less than $1 \mu \mathrm{m}$. Figure 2 shows the prototype of the stretchable strain sensor. The black part is the electrodes.

2.2. Measurement Principle. The stretchable strain sensor is assumed as a variable capacitor. Figure 3 shows the principle of the capacitance change of the strain sensor. The combination of the two electrodes and the sandwiched elastomer sheet builds a parallel plate type capacitor. The capacitance of the strain sensor depends on the area and thickness of the electrode's part (hereinafter referred to as "sensing part"). The capacitance of the strain sensor is given by

$$
C_{0}=\varepsilon_{r} \varepsilon_{0} \frac{h w}{d},
$$

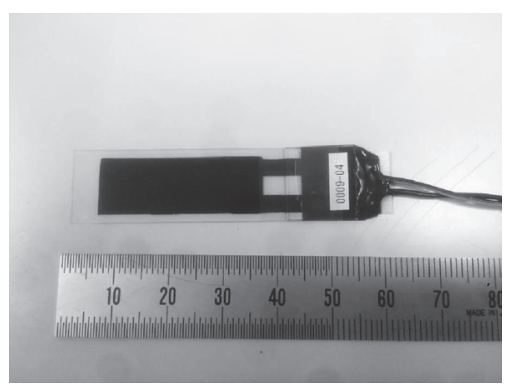

FIGURE 2: Prototype of stretchable strain sensor.

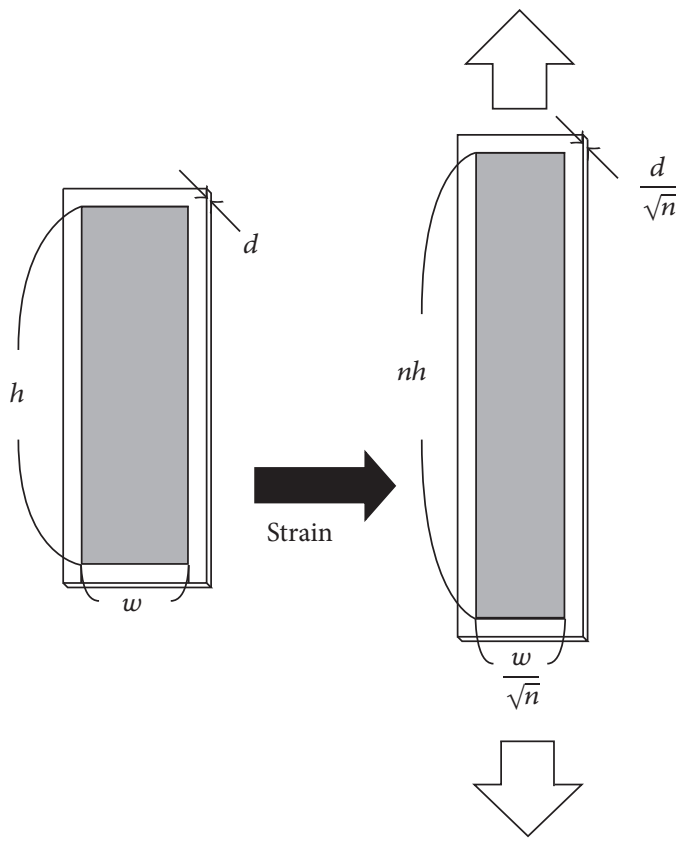

FIGURE 3: Change in size of stretchable strain sensor.

where $\varepsilon_{r}$ is the relative permittivity of the elastomer sheet, $\varepsilon_{0}$ is the permittivity in a vacuum, $h w$ is the area of the sensing part, and $d$ is the thickness of the sensing part. If Poisson's ratio of the sheet is approximately 0.5 , the volume of the sensing part is assumed to be an almost constant. When the strain sensor is stretched to $n$-fold length in a single direction, the capacitance of the strain sensor is given by

$$
C_{n}=\varepsilon_{r} \varepsilon_{0} \frac{n h w / \sqrt{n}}{d / \sqrt{n}}=n C_{0} .
$$

The length and the capacitance of the strain sensor are in a linear relationship. Therefore, the length of the strain sensor is determined based on the capacitance.

To confirm the relationship between the length and the capacitance of the strain sensor, a preliminary experiment was performed. Figure 4 shows the result of the relation between the capacitance of the strain sensor and the strain. The strain of natural length is $0 \%$. Figure 4 shows that the length and the capacitance of the strain sensor are in a linear relationship. Table 1 shows the maximal errors of the target 


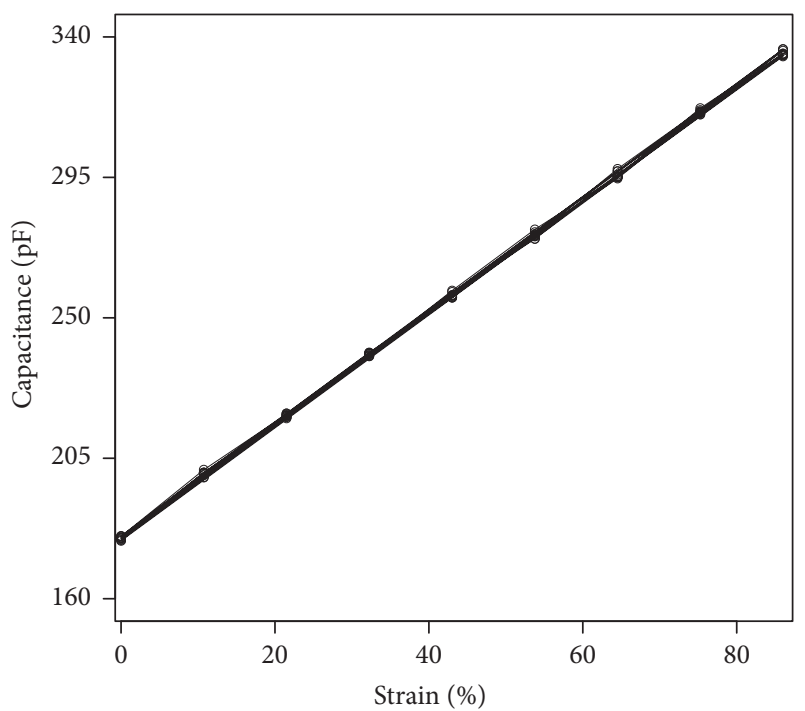

FIGURE 4: Relation between capacitance and strain.

TABLE 1: Maximal errors between target and estimate length.

\begin{tabular}{lc}
\hline & Maximal error $[\mathrm{mm}]$ \\
\hline Sensor 0 & 0.51 \\
Sensor 1 & 0.91 \\
Sensor 2 & 0.48 \\
Sensor 3 & 0.66 \\
\hline
\end{tabular}

and estimate length. The maximal error was 0.91 . It shows that the strain sensor has highly precise estimation.

\section{Rapid Prototyping Human Interface}

To make human interfaces, the stretchable strain sensors are applied to objects formed by a 3D printer. The 3D printer forms parts of the interfaces. The size of human interface depends on the size of human hands. The $3 \mathrm{D}$ printer is rapidly able to form an optimum human interface based on the size of the user's hand. Here, we make three types of interfaces: a sheet-shaped interface, a sliding lever interface, and a tilting lever interface. Both terminals of the strain sensor are attached to the parts of the interfaces. The strain sensor measures the distance between the parts as the length of itself. The output of the interface is determined based on the length of the strain sensor.

3.1. Sheet-Shaped Interface. The sheet-shaped interface comprises two frames formed by the 3D printer, one elastomer sheet, and the four strain sensors. Figure 5 illustrates the sheet-shaped interface. The frame is a $200 \mathrm{~mm}$ square and $5 \mathrm{~mm}$ in thickness. The elastomer sheet is $0.5 \mathrm{~mm}$ in thickness and is flexible and stretchable. The two frames sandwich the elastomer sheet and fix its periphery. The terminal of the strain sensor is attached to the frame, and the other terminal is attached to the center of the elastomer sheet. The four strain sensors are placed in the radial directions as shown in

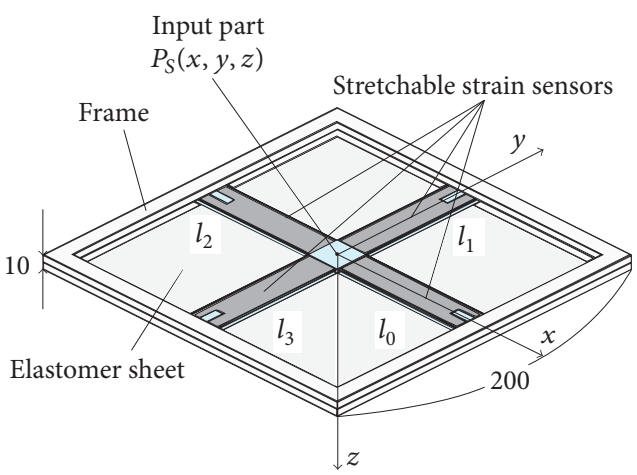

FIGURE 5: Structure of sheet-shaped interface.

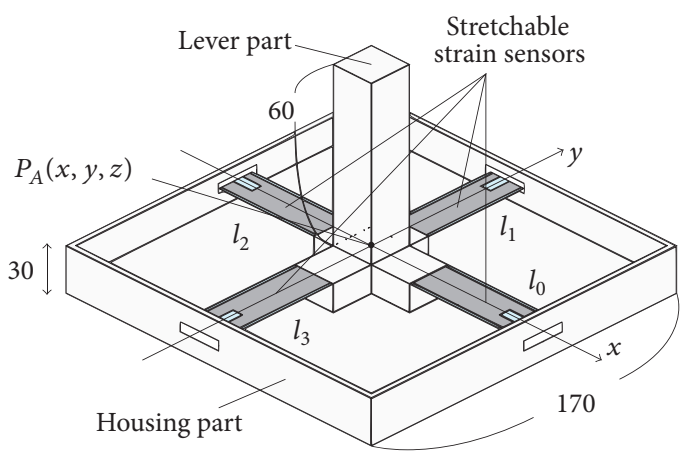

Figure 6: Structure of sliding lever interface.

Figure 5. The center of the elastomer sheet is an input part. The four lengths of the strain sensors determine the threedimensional displacement of the input part as the input of the interface. The displacement of the input part is geometrically given by

$$
\begin{aligned}
& x=\sum_{i=0}^{3} k_{x i} l_{i}^{2}+b_{x}, \\
& y=\sum_{i=0}^{3} k_{y i} l_{i}^{2}+b_{y}, \\
& z=\sum_{i=0}^{3} k_{z i} l_{i}^{2}+b_{z},
\end{aligned}
$$

where $x, y, z$ are the three-dimensional displacement of the input part; the origin is placed at the center of the elastomer sheet. $l_{i}$ is the length of the $i$ th strain sensor. $k_{x i}, k_{y i}, k_{z i}$ are coefficients for the square of the sensor's length and $b_{x}, b_{y}, b_{z}$ are constants. The coefficients and the constants are determined based on the data set composed of the threedimensional displacement of the input part and the lengths of the sensors by the least square method.

3.2. Sliding Lever Interface. The sliding lever interface comprises one lever part, one housing part, and the four strain sensors. Figure 6 shows the structure of the sliding lever interface. The lever and the housing parts are formed by the 


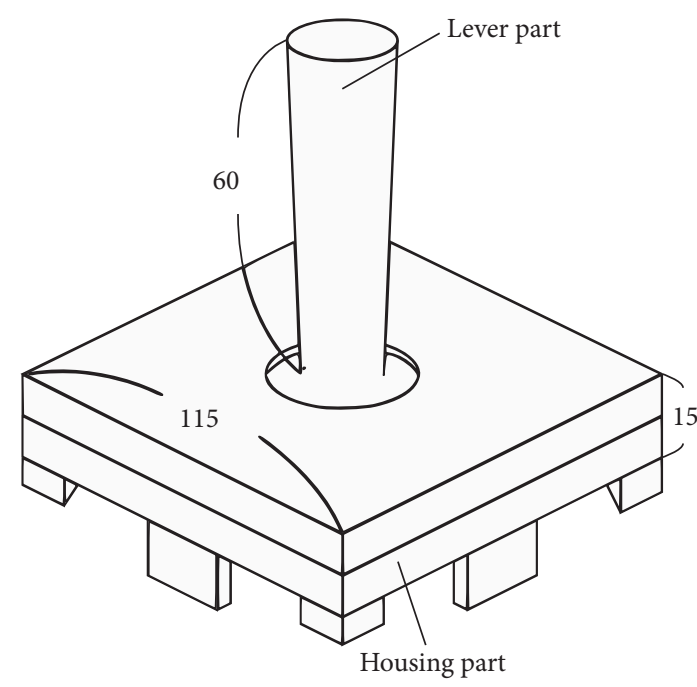

(a) Top view

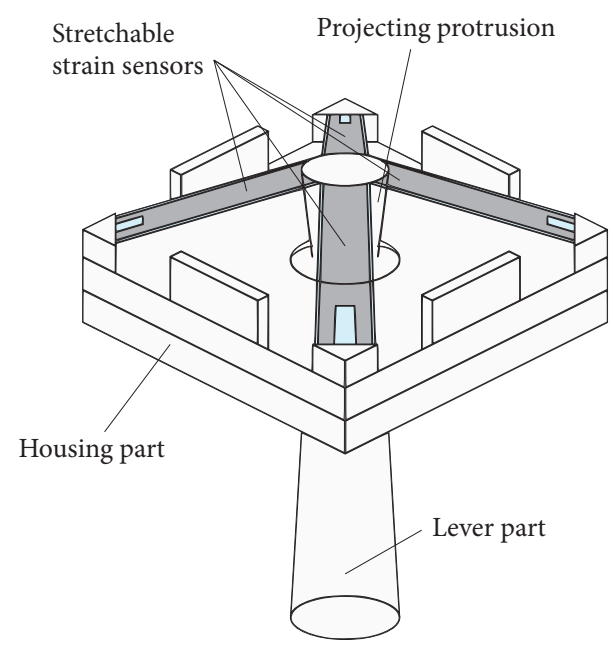

(b) Bottom view

FIgURE 7: Structure of tilting lever interface.

$3 \mathrm{D}$ printer. The housing part is $170 \mathrm{~mm}$ wide and $30 \mathrm{~mm}$ high. The height of lever part is $90 \mathrm{~mm}$. The terminal of the strain sensor is attached to the wall of the housing and the other terminal is attached to the root of the lever. The lever smoothly slides in $x$-axis and $y$-axis directions. In the same manner with the sheet-shaped interface, $x$ and $y$ are determined by (3) and (4).

3.3. Tilting Lever Interface. The tilting lever interface comprises one lever part, two housing parts, and the four strain sensors. Figure 7 shows the structure of the tilting lever interface. The lever has a sphere shape at the lower part. The two housing parts sandwich the sphere. Therefore, the sphere rotates based on the tilting angle of the lever. The opposite end of the lever has a short projecting protrusion to attach the strain sensors. The other terminal of the strain sensor is fixed at the corner of the housing part. The coordinate axes for the interface are defined as shown in Figure 8. The origin of the coordinate axes is the center of the sphere in the lever. The two angles of the interface are defined as shown in Figure 9 and are estimated as follows. The three-dimensional position of the lever's bottom end is estimated based on the lengths of the strain sensor using (3)-(5). The relationship between the two angles, $\theta$ and $\phi$, and the position of the bottom end is given by

$$
\left(\begin{array}{l}
x \\
y \\
z
\end{array}\right)=\left(\begin{array}{c}
l_{r} \sin \theta \cos \phi \\
l_{r} \sin \theta \sin \phi \\
l_{r} \cos \theta
\end{array}\right),
$$

where $l_{r}$ denotes the length between the bottom end and the center of the sphere. $\theta$ and $\phi$ are given by

$$
\theta=\cos ^{-1}\left(\frac{z}{\sqrt{x^{2}+y^{2}+z^{2}}}\right),
$$

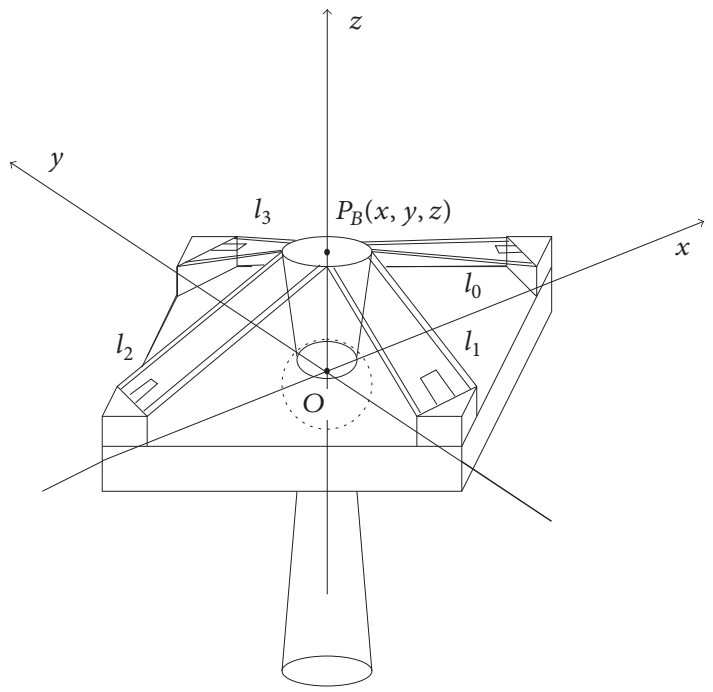

FIGURE 8: Coordinate axes of tilting lever interface.

$$
\phi=\cos ^{-1}\left(\frac{x}{\sqrt{x^{2}+y^{2}}}\right) .
$$

\section{Experiments}

4.1. Experiment Flow. Experiments were performed to evaluate the accuracy of the interfaces. Figure 10 shows the experimental apparatus and the sliding lever interface. The experimental apparatus mainly comprises a motorized stage and a PC. The PC has an analog-digital conversion board and obtains the length of the strain sensor based on the measured capacitance. The motorized stage displaces the interface 


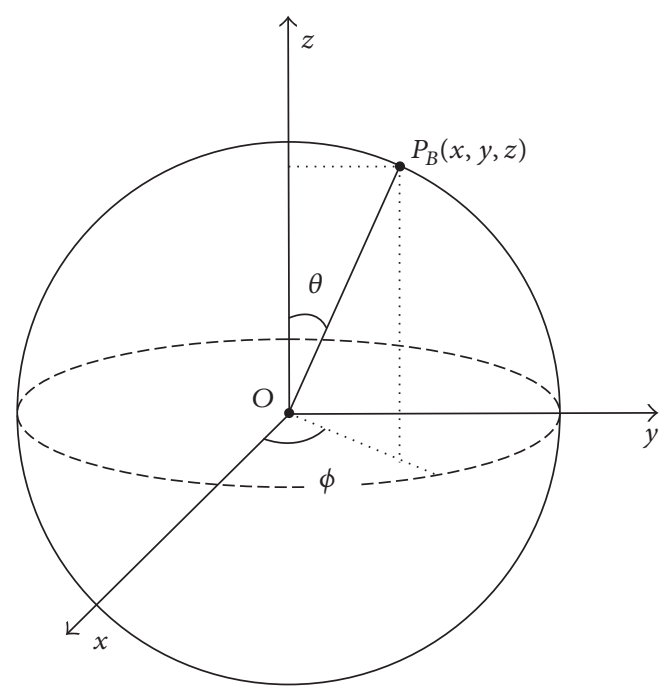

FIGURE 9: Relation between coordinate axes and angles.

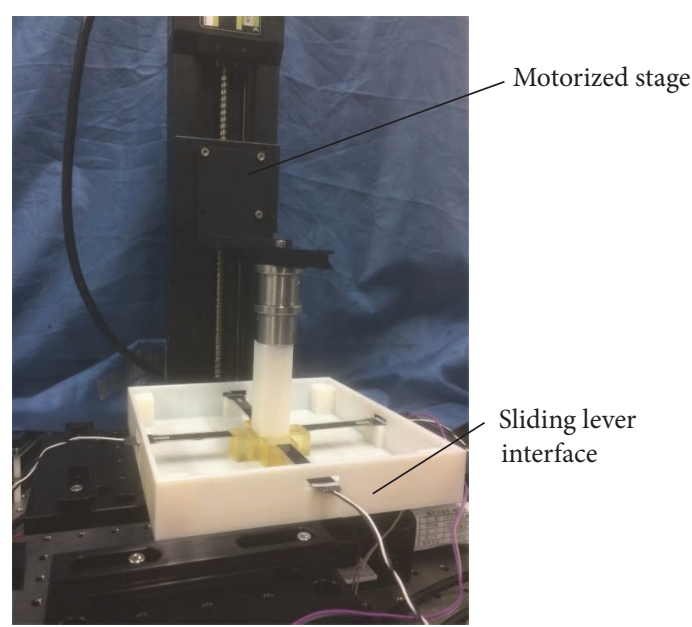

FIGURE 10: Experimental apparatus for sliding lever interface.

through the stage in $x$-axis, $y$-axis, and $z$-axis directions. The precision of the displacement is $0.01 \mathrm{~mm}$. The experimental flow is as follows. First, the data sets for calibration are obtained using the motorized stage. The coefficients and constants expressed in (3)-(5) are determined based on the data sets. Second, the motorized stage displaces the interface again. The PC estimates the outputs of the interface. The displacements of the motorized stage and the outputs of the interface are compared.

4.2. Sheet-Shaped Interface. Figure 11 shows the prototype of the sheet-shaped interface. Although the natural length of the strain sensor is $46.5 \mathrm{~mm}$, each strain sensor was attached to the elastomer sheet while being stretched to $62 \mathrm{~mm}$. The displacements were $-6.0,-3.0,0,3.0$, and $6.0 \mathrm{~mm}$ in $x$-axis and $y$-axis directions and were $11.0,16.5$, and $22.0 \mathrm{~mm}$ in $z$ axis direction. Therefore, the number of the displacements' combinations was 75 . Figures $12-14$ show the relationships between target and estimated displacements in $x$-axis and

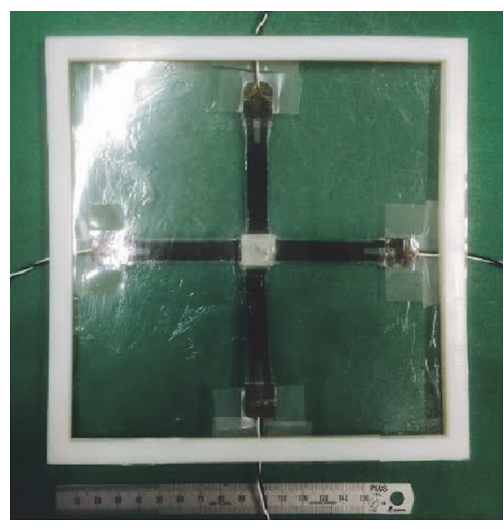

FIGURE 11: Prototype of sheet-shaped interface.

TABLE 2: Maximal errors between target and estimated displacements.

\begin{tabular}{lc}
\hline Axis & Maximal error $[\mathrm{mm}]$ \\
\hline$x(z=11)$ & 1.32 \\
$y(z=11)$ & 0.75 \\
$x(z=16.5)$ & 0.67 \\
$y(z=16.5)$ & 0.61 \\
$x(z=22)$ & 1.21 \\
$y(z=22)$ & 1.45 \\
$z$ & 3.77 \\
\hline
\end{tabular}

$y$-axis. In this paper, including graphs, target means true value measured by a motorized machine. Figure 15 shows the results in $z$-axis. In addition, Table 2 shows maximal errors in each axis.

4.3. Sliding Lever Interface. Figure 16 shows the prototype of the sliding lever interface. The strain sensors were stretched to $77 \mathrm{~mm}$ and were attached to the root of the lever and the side of the housing part. After the calibration was performed, the lever was slided $-12.0,-8.0,-4.0,0,4.0,8.0$, and $12.0 \mathrm{~mm}$ in $x$-axis and $y$-axis directions. Therefore, the number of the displacements' combinations was 49. Figures 17(a) and 17(b) show the relationships between target and estimated displacements in $x$-axis and $y$-axis. Table 3 shows maximal errors in each axis.

4.4. Tilting Lever Interface. Figure 18 shows the prototype of the tilting lever interface. The strain sensors were stretched to $70 \mathrm{~mm}$ and were attached to the bottom side of the lever part as shown in Figure $7(\mathrm{~b})$. The lever was tilted in the experiment. The angles of $\theta$ were $10^{\circ}, 20^{\circ}$, and $26^{\circ}$. The angles of $\phi$ were $0^{\circ}, 45^{\circ}, 90^{\circ}, 135^{\circ}$, and $180^{\circ}$. The number of the angles' combinations was 15 . Figure 19 shows the relationships between target and estimated angles of $\theta$ and $\phi$. Table 4 shows maximal errors of each angle. 


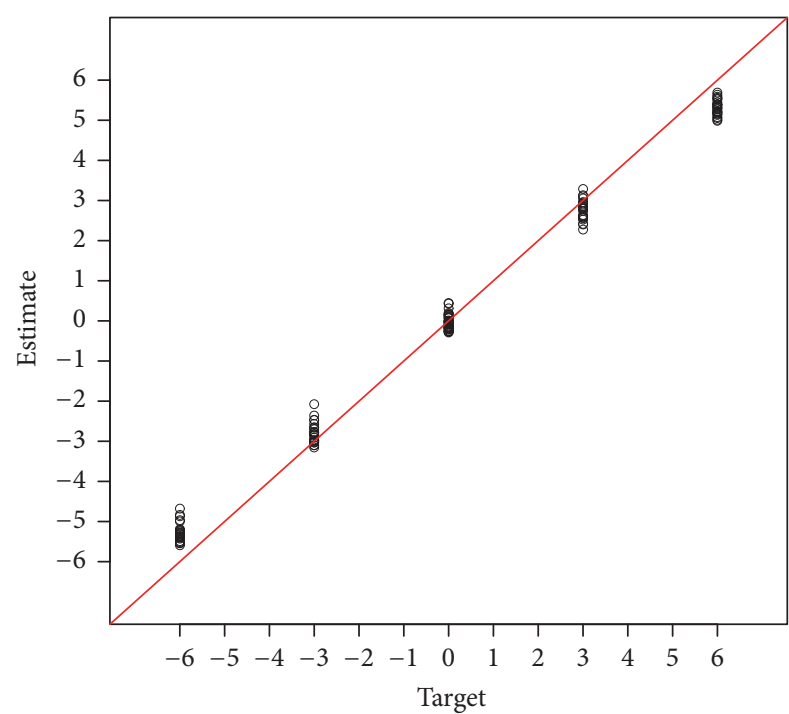

(a) $x$

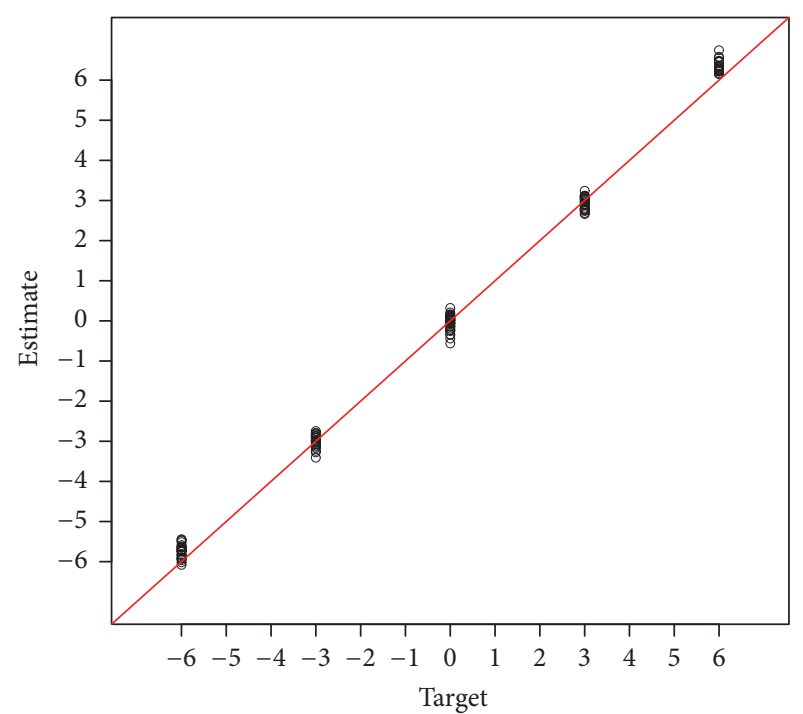

(b) $y$

FIGURE 12: Relation between target and estimated displacements under the condition of $z=11$.

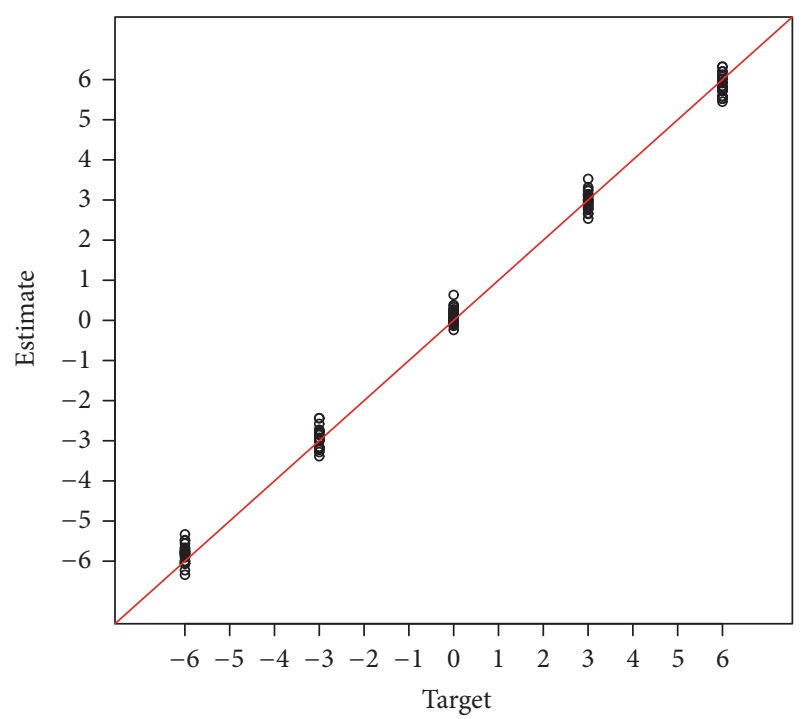

(a) $x$

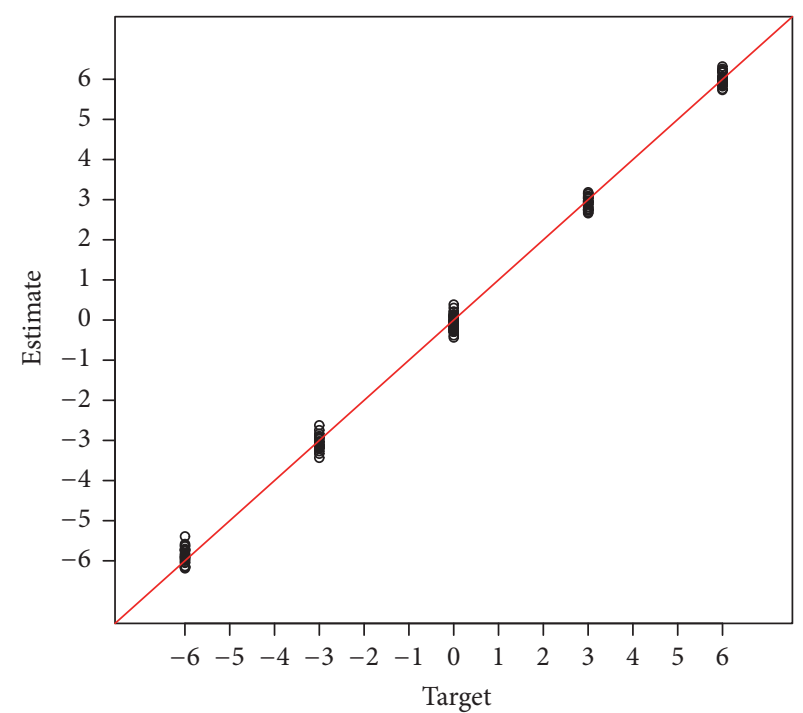

(b) $y$

FigURE 13: Relation between target and estimated displacements under the condition of $z=16.5$.

TABle 3: Maximal errors between target and estimated displacements.

\begin{tabular}{lc}
\hline Axis & Maximal error $[\mathrm{mm}]$ \\
\hline$x$ & 0.73 \\
$y$ & 1.00 \\
\hline
\end{tabular}

\section{Discussion}

The proposed interface has the sheet-shaped and the levershaped structures. They are fundamental structure for interfaces and have the ability to enhance personalized interfaces
TABLE 4: Maximal errors between target and estimate.

\begin{tabular}{lc}
\hline Angle & Maximal error $\left[^{\circ}\right]$ \\
\hline$\theta$ & 2.82 \\
$\phi$ & 10.88 \\
\hline
\end{tabular}

by changing the size and shape of the parts. Here, we discuss the accuracy of the fundamental interfaces.

The sheet-shaped interface had small maximal errors in $x$-axis and $y$-axis in Table 2 . Because the strain sensor had a measurement error within 5\%, the maximal errors in $x$ axis and $y$-axis were conceivable. On the other hand, the 


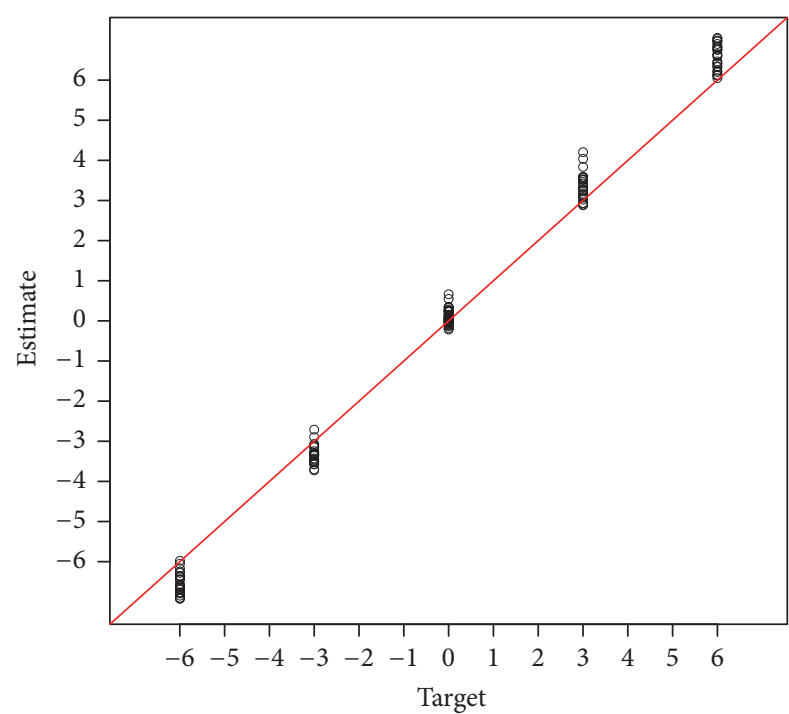

(a) $x$

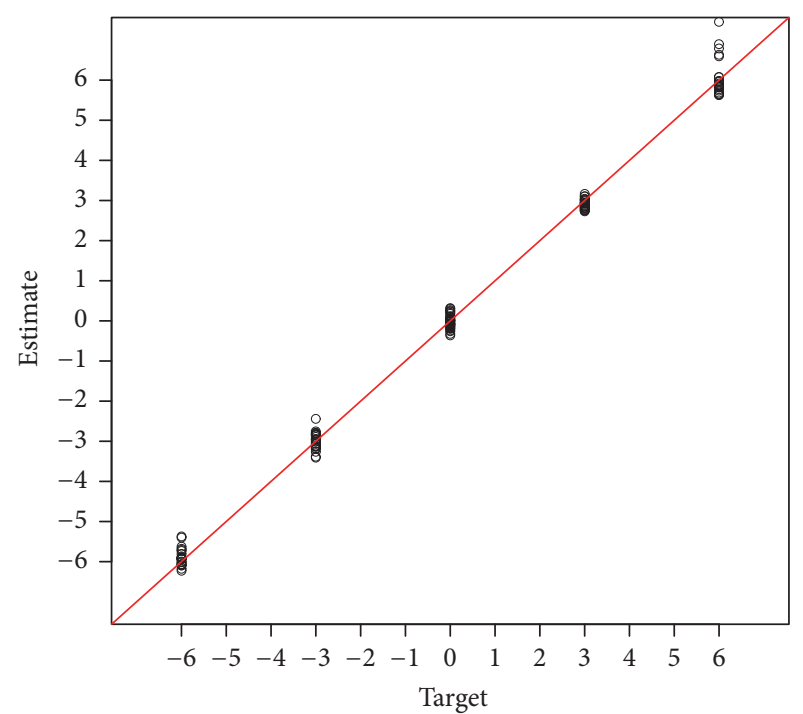

(b) $y$

FIGURE 14: Relation between target and estimated displacements under the condition of $z=22$.

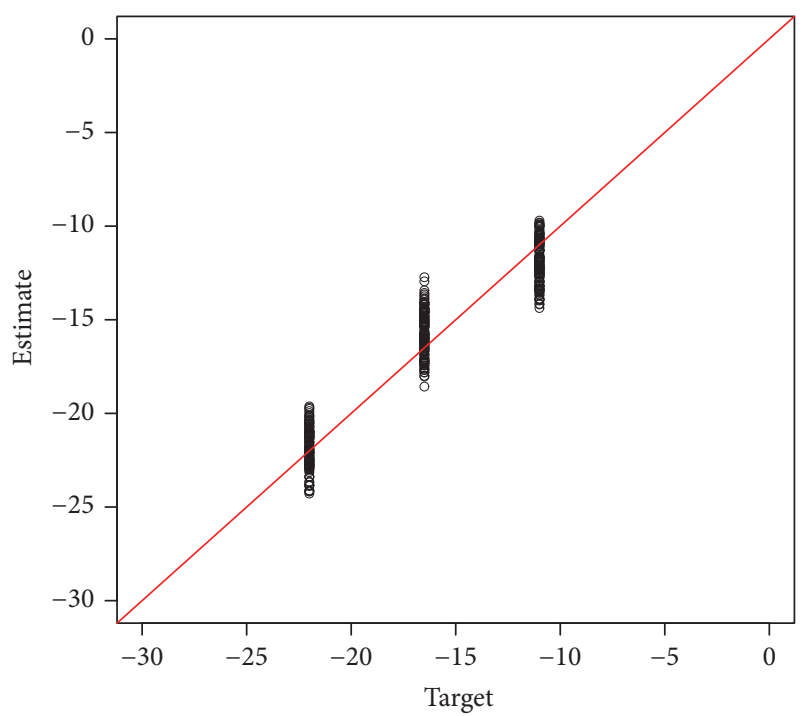

FIGURE 15: Relation between target and estimated displacements in $z$-axis.

maximal error in $z$-axis was $3.77 \mathrm{~mm}$. Although the error of $z$-axis is relatively large compared to the errors of $x$ axis and $y$-axis, the range of $z$-axis displacement is also large. We consider that a small range improves the error. The maximal errors of the sliding lever interface were as much as those of the sheet-shaped interface. The sliding lever interface used the four strain sensors for the estimate of the two-axis displacement. The number of the strain sensors was redundant and increased the accuracy of the estimate. The tilting lever interface had the large difference between the errors of $\theta$ and $\phi$. The main reason of the difference was the difference of the angle range. In Figure 19(b), the errors were almost constant in the whole range.

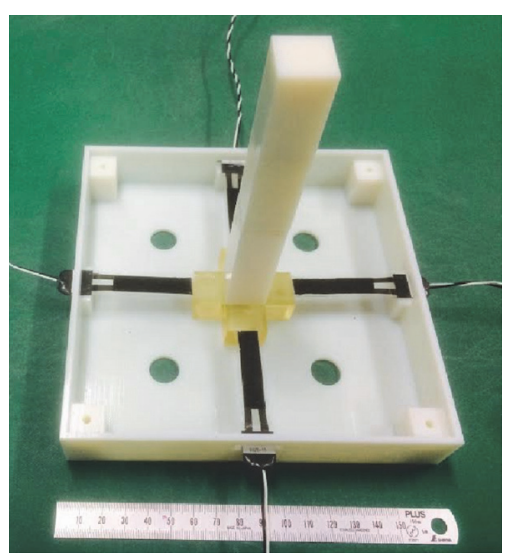

FIGURE 16: Prototype of sliding lever interface.

The results of the three interfaces showed that the errors had no bias. Actually, the outputs of the interfaces against continuous input were smooth. This characteristic is effective for interface devices. The errors of the outputs mainly depended on the measurement errors of the strain sensor, which were shown in Table 1 . The rates of their errors to the output ranges were almost constant at $10 \%$. To reduce the measurement errors, it is necessary to improve the composition and the circuit of the strain sensor.

\section{Conclusion}

The rapid prototyping human interfaces using the stretchable strain sensor were proposed. The parts of the interfaces were formed by the 3D printer, and it was easy to change their size and shape. The combinations of the parts and the four strain sensors produced the three interfaces: the sheet-shaped interface, the sliding lever interface, and the tilting lever 


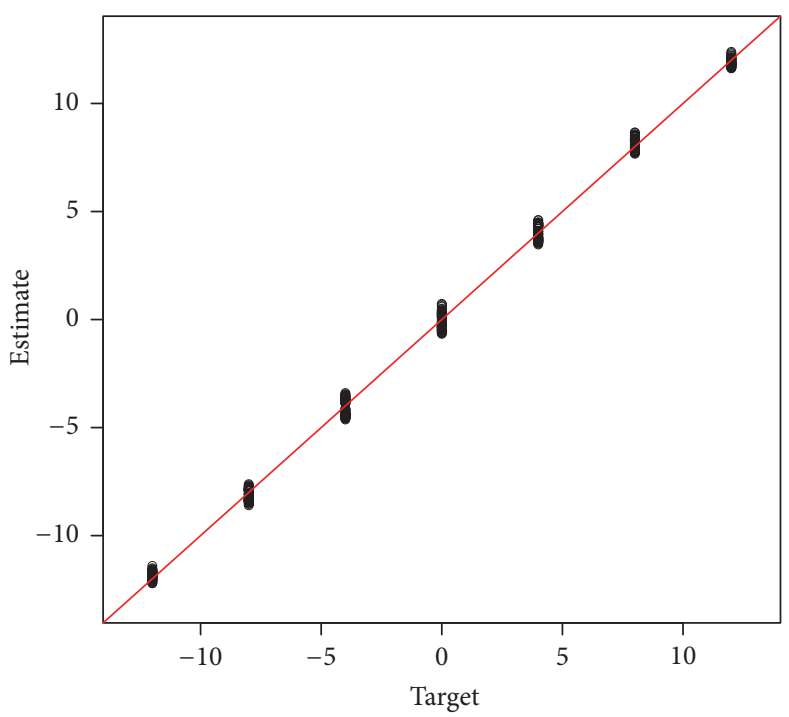

(a) $x$

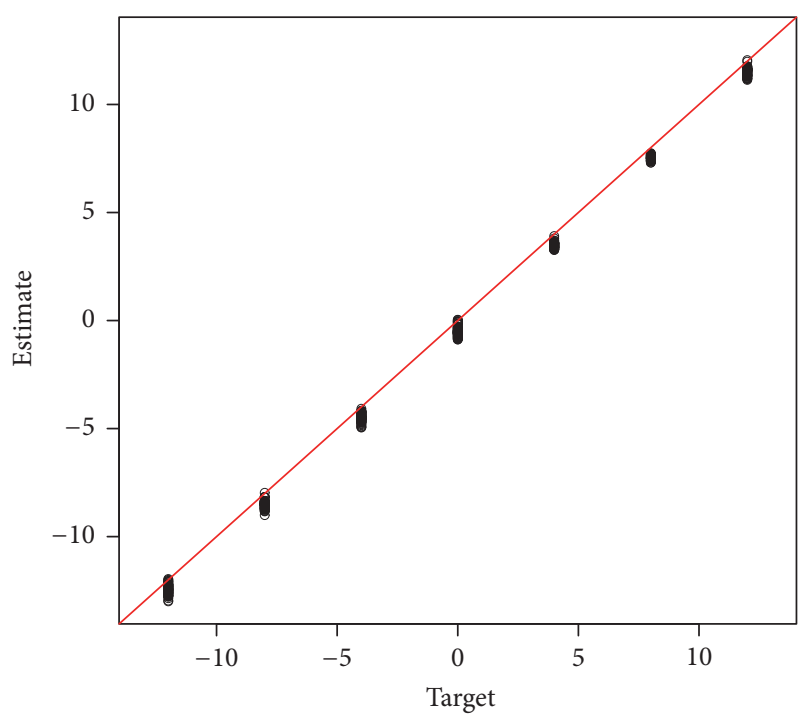

(b) $y$

FIGURE 17: Relation between target and estimated displacements of the lever sliding lever interface.

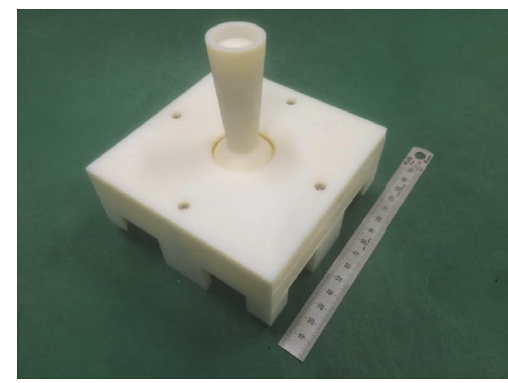

FIGURE 18: Prototype of tilting lever interface.

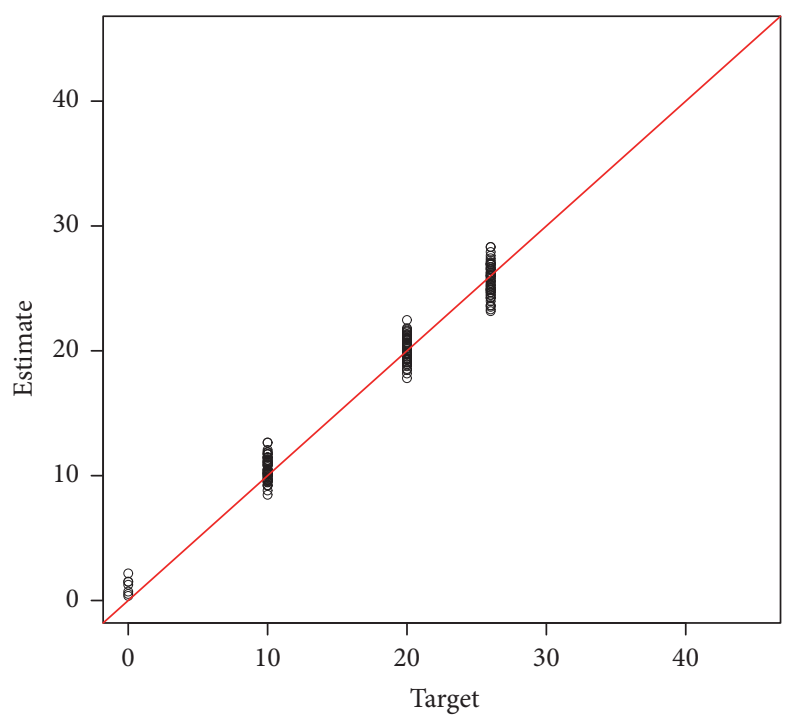

(a) $\theta$

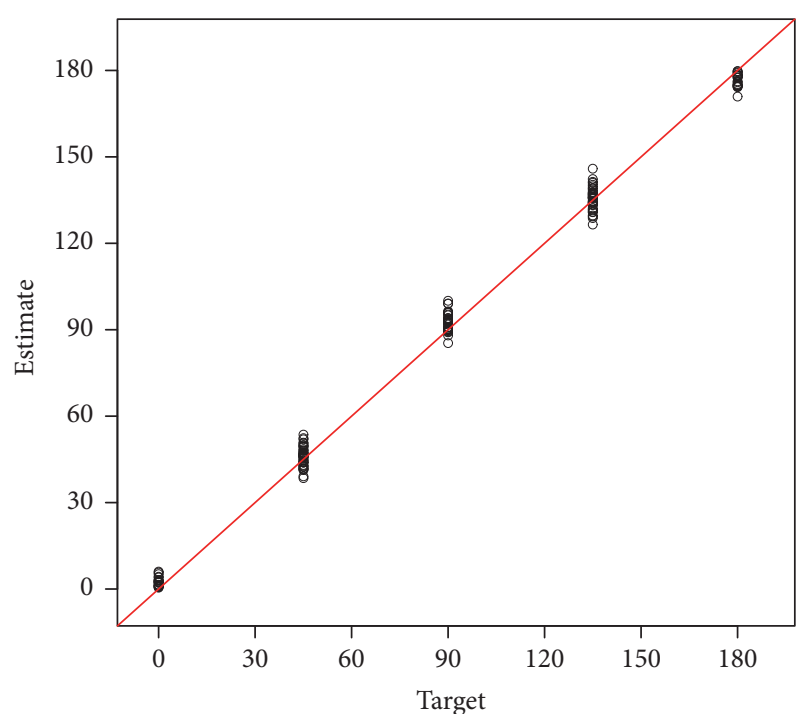

(b) $\phi$

FIGURE 19: Relation between target and estimate of tilting lever interface. 
interface. The rates of their errors to the output ranges were almost constant. The characteristic without bias is suitable for human interface devices. To improve the resolution of the interface's output, it is necessary to optimize the composition and the circuit of the strain sensor. In addition, the strain sensor is being applied to other interfaces, such as wearable interfaces.

\section{Competing Interests}

The authors declare that there are no competing interests regarding the publication of this paper.

\section{Acknowledgments}

This work was supported by SCOPE of the Ministry of Internal Affairs and Communications.

\section{References}

[1] F. Kimura and N. Yamane, "Haptic environment for designing human interface of virtual mechanical products," CIRP Annals-Manufacturing Technology, vol. 55, no. 1, pp. 127-130, 2006.

[2] S. Ha, L. Kim, S. Park, C. Jun, and H. Rho, "Virtual prototyping enhanced by a haptic interface," CIRP Annals-Manufacturing Technology, vol. 58, no. 1, pp. 135-138, 2009.

[3] Y. J. Yau, S. L. Hwang, and C. J. Chao, "Effects of input device and motion type on a cursor-positioning task," Perceptual and Motor Skills, pp. 7690, 2008.

[4] G. Turpin, J. Armstrong, P. Frost, B. Fine, C. D. Ward, and L. L. Pinnington, "Evaluation of alternative computer input devices used by people with disabilities," Journal of Medical Engineering and Technology, vol. 29, no. 3, pp. 119-129, 2005.

[5] V. Savage, C. Chang, and B. Hartmann, "Sauron: embedded single-camera sensing of printed physical user interfaces," in Proceedings of the 26th Annual ACM Symposium on User Interface Software and Technology (UIST '13), pp. 447-456, St Andrews, UK, October 2013.

[6] D.-Y. Khang, H. Jiang, Y. Huang, and J. A. Rogers, "A stretchable form of single-crystal silicon for high-performance electronics on rubber substrates," Science, vol. 311, no. 5758, pp. 208-212, 2006.

[7] D.-H. Kim and J. A. Rogers, "Stretchable electronics: materials strategies and devices," Advanced Materials, vol. 20, no. 24, pp. 4887-4892, 2008.

[8] T. Sekitani, H. Nakajima, H. Maeda et al., "Stretchable activematrix organic light-emitting diode display using printable elastic conductors," Nature Materials, vol. 8, no. 6, pp. 494-499, 2009.

[9] R. Pelrine, R. Kornbluh, Q. Pei, and J. Joseph, "High-speed electrically actuated elastomers with strain greater than $100 \%$," Science, vol. 287, no. 5454, pp. 836-839, 2000.

[10] C. Stampfer, T. Helbling, D. Obergfell et al., "Fabrication of single-walled carbon-nanotube-based pressure sensors," Nano Letters, vol. 6, no. 2, pp. 233-237, 2006.

[11] F. Carpi, R. Kornbluh, P. Sommer-Larsen, and G. Alici, "Electroactive polymer actuators as artificial muscles: are they ready for bioinspired applications?" Bioinspiration and Biomimetics, vol. 6, no. 4, Article ID 045006, 2011.
[12] Q. Gao, H. Meguro, S. Okamoto, and M. Kimura, "Flexible tactile sensor using the reversible deformation of poly(3hexylthiophene) nanofiber assemblies," Langmuir, vol. 28, no. 51, pp. 17593-17596, 2012.

[13] B. J. Munro, T. E. Campbell, G. G. Wallace, and J. R. Steele, "The intelligent knee sleeve: a wearable biofeedback device," Sensors and Actuators, B: Chemical, vol. 131, no. 2, pp. 541-547, 2008.

[14] D. J. Lipomi, M. Vosgueritchian, B. C.-K. Tee et al., "Skin-like pressure and strain sensors based on transparent elastic films of carbon nanotubes," Nature Nanotechnology, vol. 6, no. 12, pp. 788-792, 2011.

[15] M. Sato, I. Poupyrev, and C. Harrison, “Touché: enhancing touch interaction on humans, screens, liquids, and everyday objects," in Proceedings of the SIGCHI Conference on Human Factors in Computing Systems (CHI '12), pp. 483-492, Austin, Tex, USA, May 2012.

[16] H. Nakamoto, H. Ootaka, M. Tada, I. Hirata, F. Kobayashi, and F. Kojima, "Stretchable strain sensor based on areal change of carbon nanotube electrode," IEEE Sensors Journal, vol. 15, no. 4, pp. 2212-2218, 2015 . 


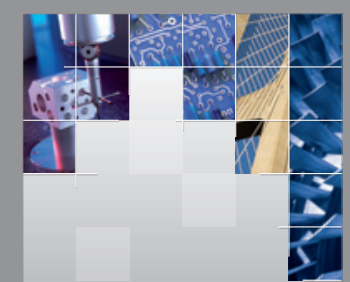

\section{Enfincering}
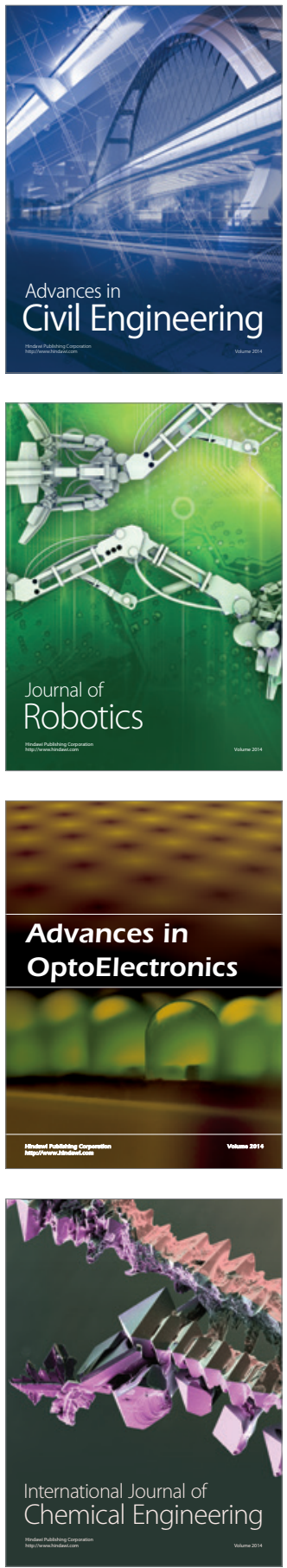

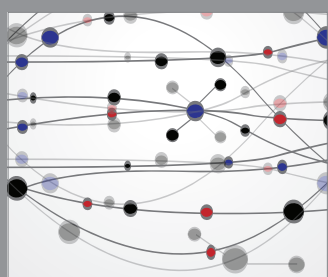

The Scientific World Journal

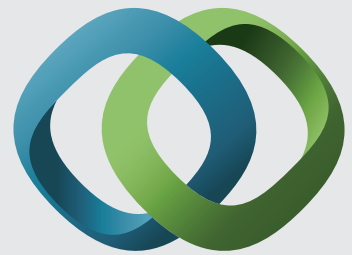

\section{Hindawi}

Submit your manuscripts at

https://www.hindawi.com
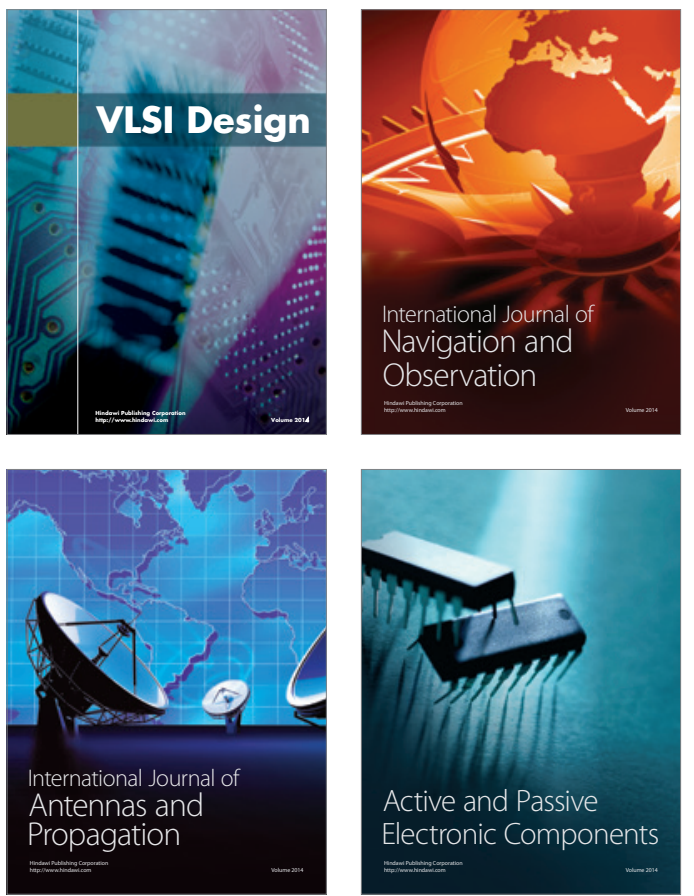
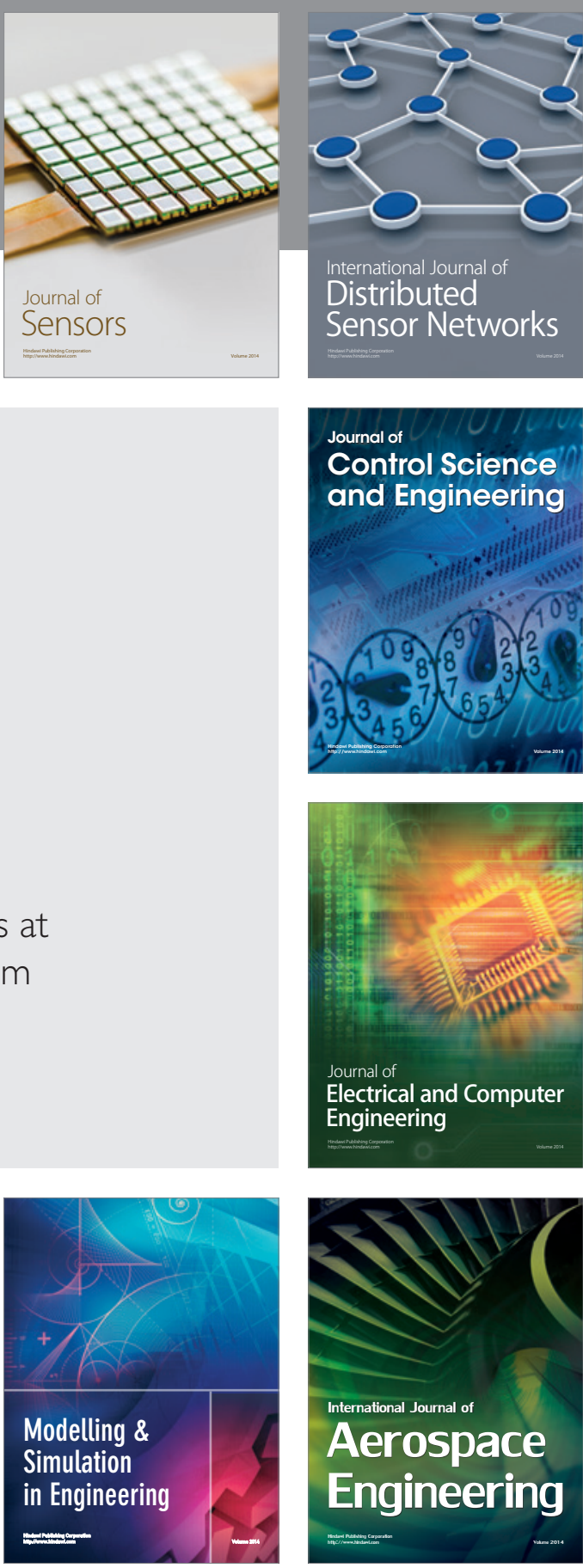

International Journal of

Distributed

Sensor Networks

$-$

Joumal of

Control Science

and Engineering
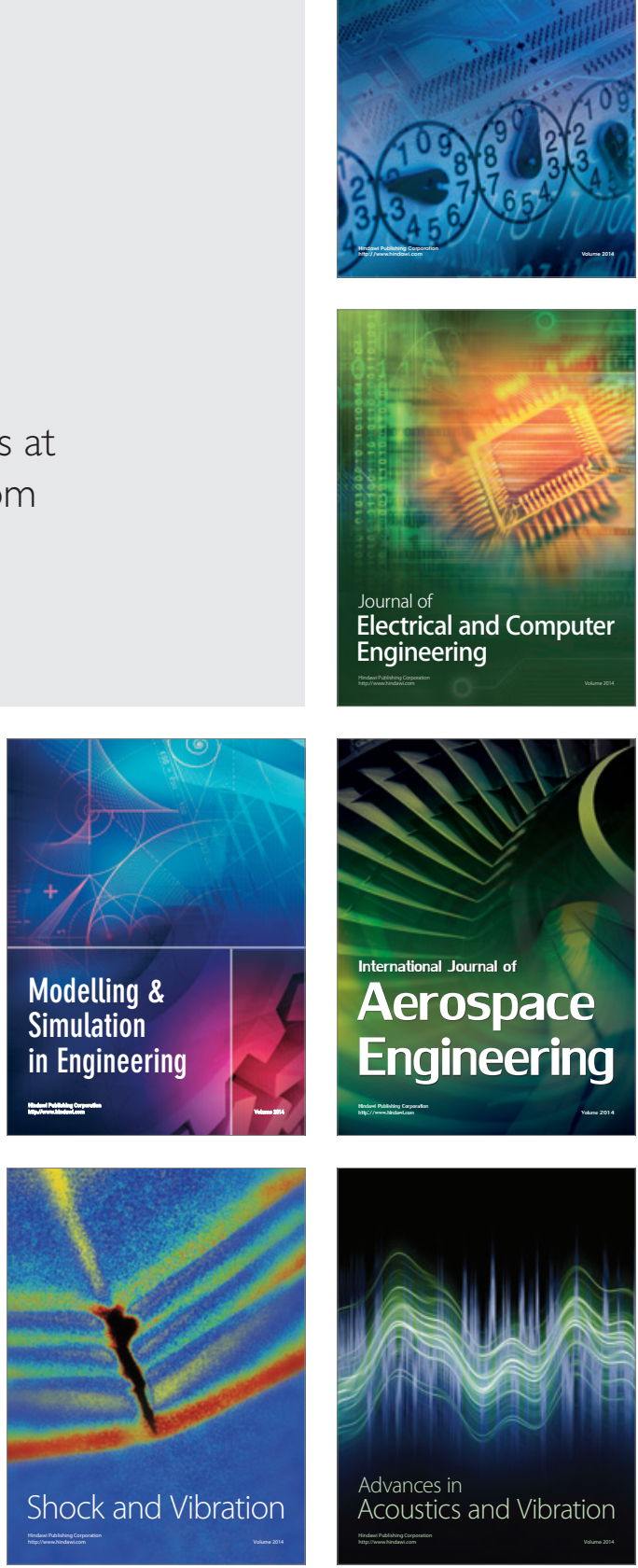